Chapman University

Chapman University Digital Commons

3-11-2019

Biomechanical Characteristics of Lumbar Manipulation Performed by Expert, Resident, and Student Physical Therapists

Joseph M. Derian

Jo Armour Smith

Yue Wang

Wilson Lam

Kornelia Kulig

Follow this and additional works at: https://digitalcommons.chapman.edu/pt_articles

Part of the Other Rehabilitation and Therapy Commons, and the Physical Therapy Commons 


\section{Biomechanical Characteristics of Lumbar Manipulation Performed by Expert, Resident, and Student Physical Therapists}

\section{Comments}

NOTICE: this is the author's version of a work that was accepted for publication in Musculoskeletal Science and Practice. Changes resulting from the publishing process, such as peer review, editing, corrections, structural formatting, and other quality control mechanisms may not be reflected in this document. Changes may have been made to this work since it was submitted for publication. A definitive version was subsequently published in Musculoskeletal Science and Practice, volume 48, in 2020.

https://doi.org/10.1016/j.msksp.2020.102150

The Creative Commons license below applies only to this version of the article.

\section{Creative Commons License}

\section{(c) (1) (9)}

This work is licensed under a Creative Commons Attribution-Noncommercial-No Derivative Works 4.0 License.

\section{Copyright}

Elsevier 


\section{KEYWORDS}

Education, Ground reaction force, Kinematics, Manual therapy/spine, Thrust 


\section{INTRODUCTION}

Low back pain is one of the most common musculoskeletal pain syndromes in the United States. In 2015, the global point prevalence was estimated at $7.3 \%$, meaning that 540 million people are affected by low back pain at any one time. ${ }^{23}$ Thus, development of effective treatments for low back pain, and the proper delivery of these treatments, is of utmost importance. Spinal manipulation, defined as the application of rapid movement to vertebral segments, is an effective treatment for low back pain and is a first-line intervention in primary care/direct access settings. $5,11,14,15,19,20$

(n)

Despite its importance, the optimal technique for performing the motor skill of lumbar manipulation has not been identified. Researchers have investigated the forces applied to the patient during manipulative techniques. ${ }^{2,4,6,7,8,9,21,22}$ The movements that the practitioner makes in order to generate these forces are less well understood.

Therefore, current teaching of this manual skill to entry-level and post-professional clinicians is neither standardized nor based upon evidence. A recent Delphi study found that practitioners who teach side-lying lumbar manipulation believe that maintaining close contact with the patient, generating force through body and legs, dropping the body downwards, and providing a "short-amplitude high-velocity" thrust are important characteristics of clinician movement during manipulation. ${ }^{18}$ This suggests that linear motion of the center of mass, pelvis kinematics, and ground reaction forces may be important biomechanical features of manipulation performance. Center of mass mechanics provide an estimation of total body motion during the thrust while measurement of angular and linear pelvis kinematics may help to demonstrate how forces are generated while contact with the patient is maintained. Vertical and horizontal 
25 ground reaction forces provide a measure of how the interaction with the ground

26 through the feet is modulated by the clinician to generate motion.

28 The purpose of this study was to identify primary features of ground reaction forces, center of mass mechanics, and pelvic kinematics during lumbar manipulation and to determine which of these features distinguish experts from less experienced practitioners. We hypothesized that expert performance of side-lying lumbar manipulation is characterized by increased rate of ground reaction force modulation,

33 faster pelvic movement, and greater center of mass momentum of the practitioner. statement prior to inclusion in the study.

\section{Participants}

Practitioners

42 Four groups of practitioners were recruited via email through professional networks of

43 the investigators and the student body of the XXX residency and entry-level physical

44 therapy programs. Practitioners were grouped into four categories: experts, residents,

45 entry-level Doctor of Physical Therapy (DPT) students in their final (third) year of

46 training, and DPT students in their first year of training. Experts were eligible for

47 inclusion if they had been practicing for a minimum of 10 years and were either

48 frequently performing the side lying lumbar manipulation in clinical practice or teaching 
manipulation techniques, including side-lying lumbar manipulation, to post-graduate physical therapists. Residents were recruited from current Orthopedic and Sports Physical Therapy residency cohorts. All residents were licensed physical therapists who had recently graduated from an APTA credentialed entry-level DPT program. Students were recruited from current first year and third year DPT cohorts at the same institution. To help homogenize body type and size, only male participants were recruited.

\section{Patient-Models}

Patient-models had to be between the ages of 18 and 35 and have at least one hypomobile lumbar spine segment, assessed via prone posterior-to-anterior glide. Although this method has moderate to poor inter-tester reliability it is also a very widely used method of assessing spinal stiffness and is part of a clinical prediction rule for those likely to benefit from spinal manipulation. ${ }^{5,16}$ Exclusion criteria for patient-models were life history of low back pain and contraindications/risk factors to manipulation (known presence of a disc herniation, known pars defect, Beighton score greater than 4 , active infection, cancer history or rheumatoid arthritis). To help homogenize body type and size and reduce risk of side effects, all male patient-models were used. ${ }^{1}$

\section{Data Collection Procedure}

Models lay in the right lateral recumbent position on a high-low table in front of two force plates (AMTI OR-6, Watertown, MA) (Figure 1). Practitioners stood with one foot on each force plate facing the model. Vertical and horizontal ground reaction force (GRF) data were sampled at $1600 \mathrm{~Hz}$. The practitioners were instrumented with $14 \mathrm{~mm}$ retroreflective markers placed on the skin overlying the L5-S1 spinous process interspace and the iliac crests and on the greater trochanters. Motion capture data were collected 
using an 11-camera motion capture system (Qualisys Oqus System, Qualisys AB, Gothenburg, Sweden) sampling at $200 \mathrm{~Hz}$. First, a calibration trial was collected with the participant standing still to establish the dimensions of the pelvic segment. The greater trochanter markers were then removed and pelvic motion during the manipulation was tracked using the L5-S1 and iliac markers. Ground reaction force and motion capture data were digitally synched (Qualisys Track Manager, Qualisys AB, Gothenburg, Sweden). Practitioners completed two manipulations on one model, and two on another model for a total of four manipulations per practitioner. Each manipulation was separated by at least 30 minutes to allow for absorption of synovial joint gasses produced by potential cavitation. ${ }^{3}$
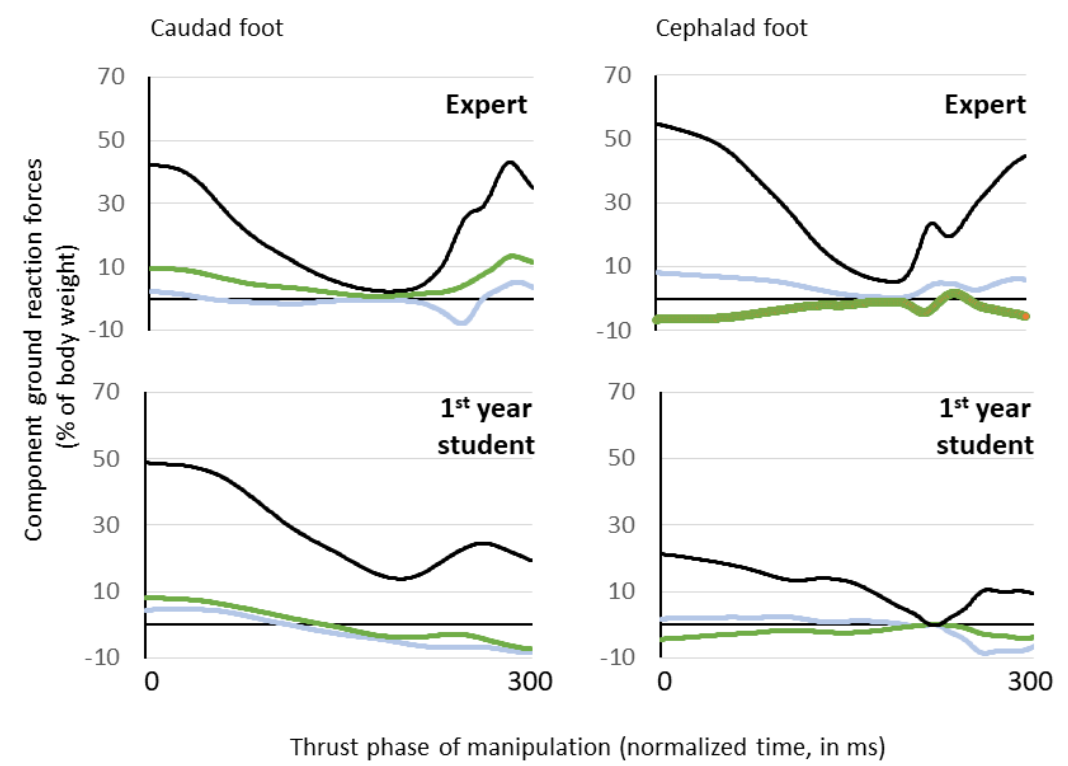

Force plate coordinate system
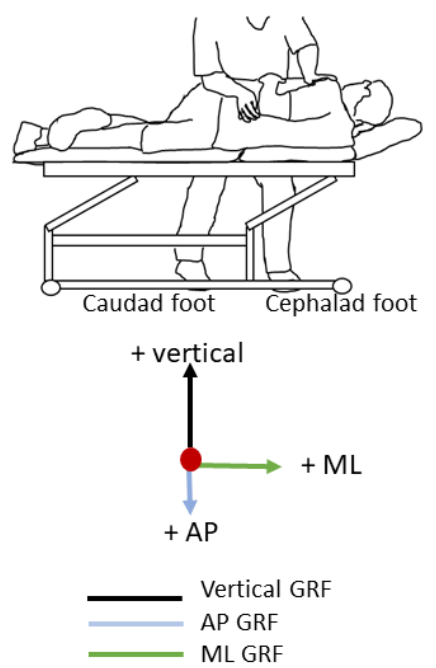

Thrust phase of manipulation (normalized time, in ms)

FIGURE 1. Exemplar vertical and horizontal ground reaction force data from one expert (top) and one first year student (bottom). The force plate coordinate system is shown, with the positive mediolateral (ML), anteroposterior (AP) and vertical directions indicated relative to the practitioner. The caudad and cephalad foot was defined in reference to the position of the patient-model. The thrust phase of the manipulation was defined as: the moment when vertical GRF under one or both feet peaked prior to rapidly decreasing until the lowest height of the L5S1 marker. 
Data Processing

91 Kinetic analysis

92 Ground reaction force (GRF) data were low-pass filtered at $50 \mathrm{~Hz}$. For each trial, onset of the thrust phase of the manipulation was defined as the moment when vertical GRF under one or both feet peaked prior to rapidly decreasing (Figure 2). This event indicates the start of the thrust as it is the beginning of a sharp drop in GRF associated

97 immediately precedes the start of the downward movement of the practitioner's center of mass. The end of the thrust phase of the manipulation was defined as the moment when the L5-S1 marker (a proxy for the center of mass) reached its lowest point (Figure 2).The completion of the thrust was defined in this way as practicing clinicians agree that the side-lying lumbar manipulation is a primarily downward body movement. ${ }^{18}$ Vertical and horizontal GRF data were normalized to body weight. This removed the potentially confounding influence of practitioner weight from the kinetic analyses. The following variables were calculated for the cephalad and caudad foot: vertical GRF (GRFv), anteroposterior GRF (GRF $\left.{ }_{A P}\right)$ and mediolateral GRF (GRF $\left.{ }_{M L}\right)$ at the beginning of the thrust phase; minimum (lowest point) of the GRFv during the thrust phase; rate of

107 GRFv decrease during the thrust phase (peak slope of the normalized GRFv/time curve 108 from start of thrust phase to minimum GRFv). Cephalad and caudad were defined 109 relative to the model's position (Figure 1). 


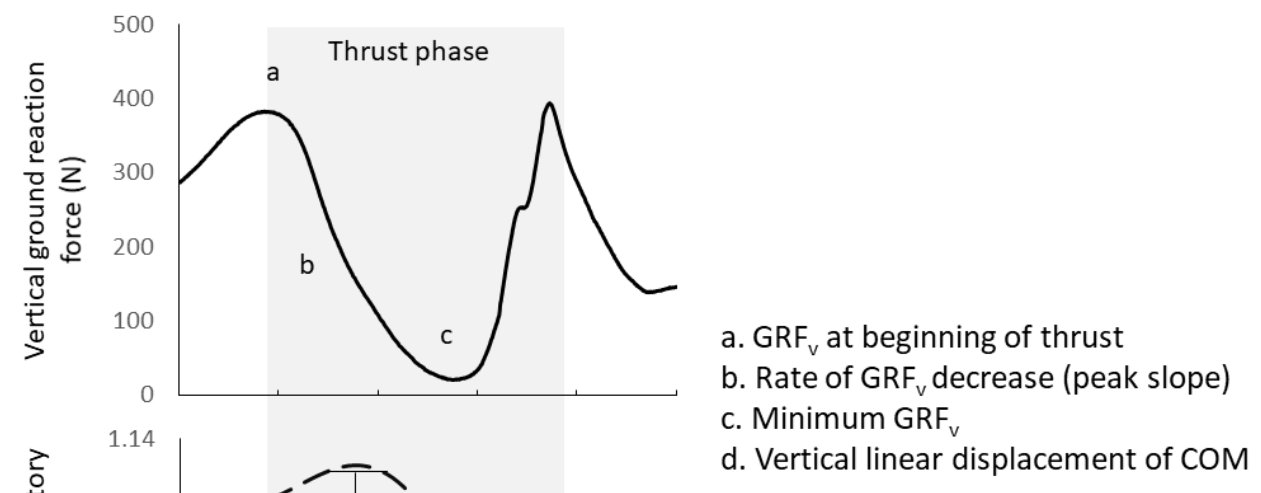

111 FIGURE 2. Exemplar vertical ground reaction force data (top) and center of mass vertical

112 trajectory data (bottom) from a single expert participant. The thrust phase of the manipulation is 113 defined as starting at the peak of the vertical ground reaction (GRF $\mathbf{F}_{\mathbf{v}}$ ) under one or both feet 114 prior to the thrust, ending at the lowest point of the center of mass trajectory after the thrust. 115 Selected kinetic and kinematic variables are indicated.

117 Force-force analysis

118 Coordination of modulation of GRFv between the two feet was calculated utilizing a 119 modified vector coding method. ${ }^{17}$ Vector coding is used to identify the relationship 120 between the magnitude of two changing variables over time. In this case, it was the 121 GRFv of each foot. The vector coding method can be visualized using a force-force 122 scatterplot with the x-axis representing the magnitude of the caudad foot GRFv and the $123 \mathrm{y}$-axis representing the magnitude of the cephalad foot GRFv (Figure 3). Each point on 124 the graph represents these values at one time point during the thrust. The angle (drawn 125 from the right horizontal) from one time point to the next is defined as the "coupling 126 angle-The coupling angle at each time point is used to define the pattern of 
127 coordination of GRFv between the feet at that time point as shown in Figure 3. An

128 inphase decreasing coordination pattern occurs when the cephalad and caudad foot

129 GRFv decrease synchronously at a similar rate, whereas a cephalad foot decreasing

130 pattern occurs when the cephalad foot GRFv is decreasing more rapidly than caudad

131 foot GRFv, and vice versa for caudad foot decreasing pattern. The time spent in each

132 coordination pattern was expressed for each individual as a percentage of the total

133 thrust time. Full details and equations for the vector coding methodology are provided in

134 Appendix A. ${ }^{17}$

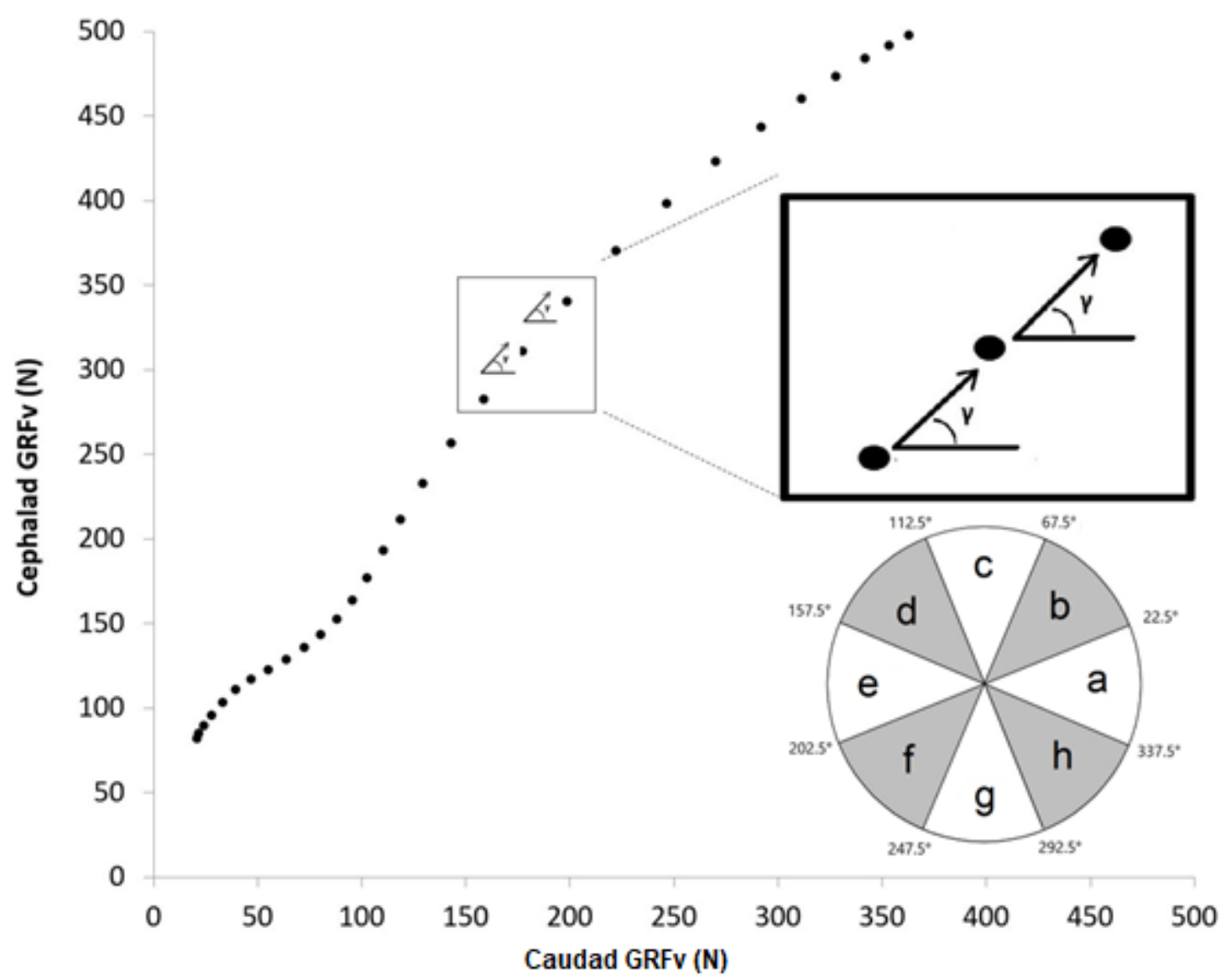

136 FIGURE 3. Example force-force scatterplot comparing change in GRFv in each foot through 137 time. Top inset: coupling angle determined by vector orientation between two adjacent data 138 points in time relative to the right horizontal. Bottom inset: Coupling angle chart. Key to patterns 139 of GRFv coordination: a; Caudad GRFv increasing (coupling angle between 337.5 degrees and 
22.5 degrees), b; Cephalad and caudad GRFv increasing (inphase increasing), c; Cephalad GRFv increasing, d; Cephalad GRFv increasing \& caudad GRFv decreasing (antiphase), e; Caudad GRFv decreasing, f; Cephalad and caudad GRFv decreasing (inphase decreasing), g; Cephalad GRFv decreasing, h; Cephalad GRFv decreasing and caudad GRFv increasing (antiphase).

\section{Kinematic analysis}

A 3-dimensional model of the pelvis was constructed from the static calibration trial using the markers on the greater trochanters, iliac crests and L5-S1. A virtual coordinate system was calculated that translated and rotated the global laboratory coordinate system to the position of the practitioner's pelvis at the start of the thrust and all motion was referenced to this starting position. The following variables were calculated during the thrust (from the highest point of the L5-S1 marker to the lowest point of the marker): peak angular velocity of the pelvis in the sagittal, frontal and transverse planes and peak vertical linear velocity of the pelvis.

Vertical linear displacement and acceleration of the center of mass (COM) was approximated by tracking the linear motion of the marker placed on the L5-S1 marker. ${ }^{13}$ COM variables were normalized to the height of the COM for each individual during the standing static calibration trial.

\section{Statistical Analysis}

All data were tested for normality of distribution. Variables that were not normally distributed were log-transformed.

\section{Group comparisons}


165 Participant age was compared between groups utilizing the Mann Whitney $U$ test. All 166 other group comparisons were made with one-way analysis of variance (ANOVA) (four

167 levels; experts, residents, third year students, first year students). Pairwise post-hoc 168 group comparisons were made for variables with a significant main effect of group. To 169 account for unequal sample size and reduce family-wise Type 1 error rate, the 170 conservative Dunnett's T3 test was utilized for post-hoc comparisons. ${ }^{10}$ Estimates of

171 effect sizes were calculated with an unbiased Cohen's $d$, with correction for small

172 sample size $\left(d_{u n b}\right) \cdot{ }^{12} 0.8$ indicates a large effect size, 0.5 a medium effect size and 0.3 a 173 small effect size.

175 Regression

176 Multiple regression was performed to explore the variables that contributed to 177 manipulation performance in addition to years of experience. The metric of manipulation 178 performance was defined as the biomechanical variable that best discriminated 179 between groups. First, bivariate correlation analyses were conducted to identify 180 potential kinematic and kinetic predictor variables. For the regression model, years of 181 experience was entered first. Then a forward stepwise approach was used to determine 182 which other predictor variables significantly contributed to variance in manipulation 183 performance ( $\alpha_{\text {enter }}=.05$ and $\alpha_{\text {exit }}=.10$, IBM SPSS Statistics, Version 25). 


\begin{tabular}{|cccccc|}
\hline & Experts $^{*}$ & Residents $^{\dagger}$ & $\begin{array}{l}\text { Third year } \\
\text { students }^{\ddagger}\end{array}$ & $\begin{array}{l}\text { First year } \\
\text { students }^{\S}\end{array}$ & p value \\
\hline Age (years) & $66.30(4.10)$ & $26.63(1.43)$ & $28.77(5.33)$ & $23.93(2.15)$ & $<0.05$ \\
\hline Height (m) & $1.81(0.06)$ & $1.80(0.07)$ & $1.82(0.07)$ & $1.81(0.08)$ & 0.067 \\
\hline Mass (kg) & $96.05(9.81)$ & $83.66(11.78)$ & $83.95(15.25)$ & $76.49(7.00)$ & 0.029 \\
\hline $\begin{array}{c}\text { Manipulation } \\
\text { experience (years) }\end{array}$ & $44.50(5.20)$ & $3.22(0.75)$ & $2.15(0.36)$ & $0.33(0.00)$ & $<0.0001$ \\
\hline
\end{tabular}

$189 P$ value indicates significance of Mann-Whitney $U$ Tests (age) and ANOVA $F$ tests for main 190 effect of group (height, mass and manipulation experience)

TABLE 1. Participant demographics/morphometrics

\section{RESULTS}

\section{Demographics}

197 Demographic information for all participants is shown in Table 1. There was no difference in height between groups. There was a group difference in mass, with experts tending toward being significantly heavier than first year students (post-hoc $p=$ .086). As expected, experts were significantly older than the other three groups $(p<$

201.005 for all post-hoc comparisons). Similarly, experts had significantly greater

202 manipulation experience than all other groups ( $p<.001$ for all post-hoc comparisons).

203 Residents had greater experience than both student groups and the third-year students 204 had more experience than the first-year students ( $p<.01$ for both comparisons). 


\section{Kinematic analysis}

206 See Appendix 2 for all between group omnibus (F-test) statistics and Table 2 for post-

207 hoc group comparisons. Two participants in the first-year student group were excluded

208 from the kinematic analyses due to occlusion of iliac crest markers during the

209 manipulation.

210

211 Pelvis motion - angular
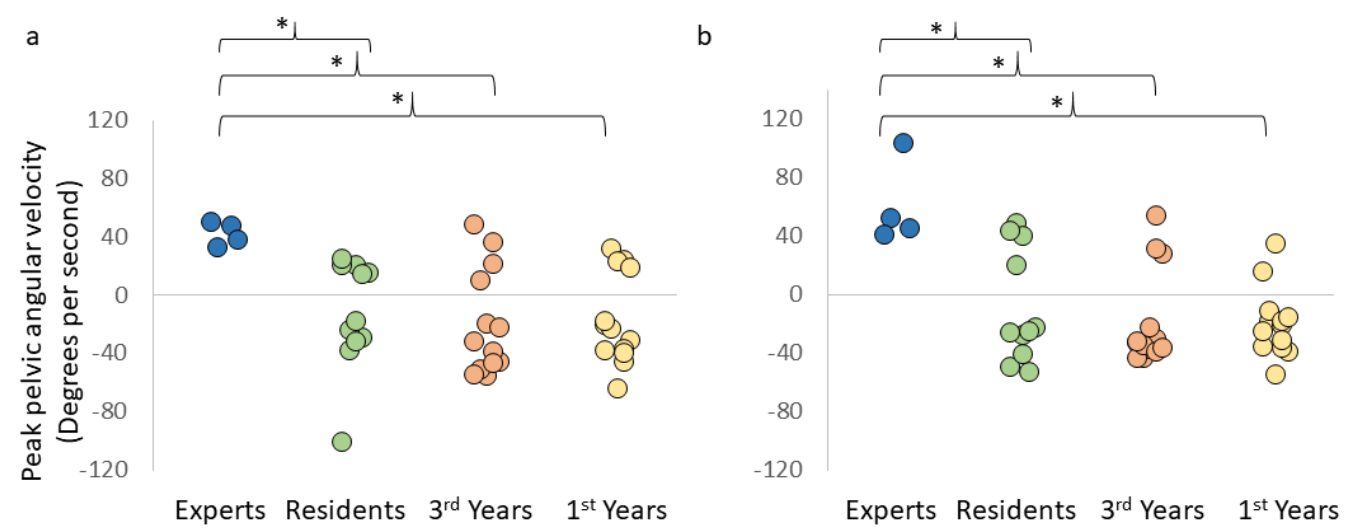

212

213 FIGURE 4. Peak pelvic angular velocity for each individual in all groups. a. Frontal

214 plane. Positive angular velocity indicates caudad (right) side flexion b. Transverse

215 plane. Positive angular velocity indicates cephalad (left) rotation. Asterisks indicates

216 significant post-hoc comparison between groups.

220 Peak angular velocity of the pelvis in the sagittal plane did not differ between groups.

221 However, there was a group difference in velocity of pelvis motion in the frontal plane. 
222 Experts had greater peak angular velocity than all other groups and all of the experts 223 demonstrated peak angular velocity in the direction of caudad side flexion (right side of

224 the pelvis tilting downward) whereas on average all of the other groups demonstrated 225 peak pelvic angular velocity in the direction of cephalad side flexion (Figure 4a. Table 226 2). There was also a difference between groups for peak angular velocity of the pelvis in 227 the transverse plane. Experts had greater peak angular velocity than all other groups 228 and experts all rotated the pelvis cephalad (toward the model's head) whereas on 229 average the other groups rotated toward the caudad side (Figure 4b. Table 2).

230 Pelvis motion - linear

231 Peak downward velocity of the pelvis was significantly different between groups Experts 232 had greater downward linear velocity than third year students and first year students.

233 Residents had greater downward linear velocity than first year students (Figure 5, Table $2342)$. 


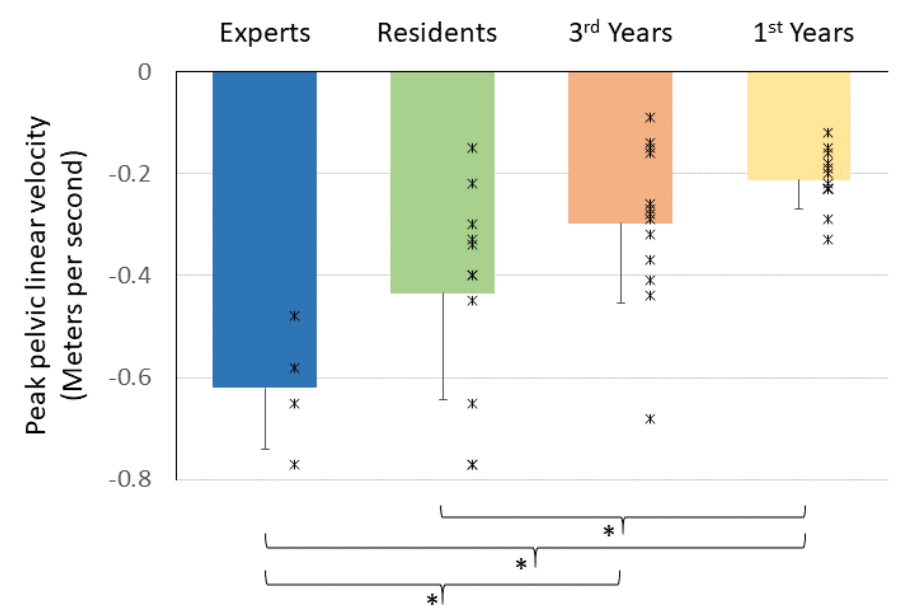

238 FIGURE 5. Peak downward linear velocity. Error bars represent group standard 239 deviations. Crosses are individual data points for each group and asterisks indicate 240 significant post-hoc comparisons between groups.

244 Center of mass motion

245 There was a significant difference between groups for the vertical displacement of the

246 COM. Experts had greater displacement than first year students (Table 2). Vertical

247 (downward) acceleration of the COM also differed significantly by experience. Experts

248 and third year students had significantly greater downward COM acceleration than first 249 year students (Table 2). 


\begin{tabular}{|lcccccc|}
\hline & $\begin{array}{c}\text { Experts: } \\
\text { Residents }\end{array}$ & $\begin{array}{c}\text { Experts: } \\
\text { third years }\end{array}$ & $\begin{array}{c}\text { Experts: } \\
\text { first years }\end{array}$ & $\begin{array}{c}\text { Residents: } \\
\text { third years }\end{array}$ & $\begin{array}{c}\text { Residents: } \\
\text { first years }\end{array}$ & $\begin{array}{c}\text { third years: } \\
\text { first years }\end{array}$ \\
\hline Pelvis peak frontal & $\mathbf{0 . 0 0 4}$ & $\mathbf{0 . 0 0 0}$ & $\mathbf{0 . 0 0 0}$ & 0.999 & 1.000 & 1.000 \\
AV & $\mathbf{1 . 4 1 1}$ & $\mathbf{1 . 6 0 1}$ & $\mathbf{1 . 5 6 8}$ & 0.087 & 0.009 & 0.058 \\
\hline Pelvis peak & $\mathbf{0 . 0 3 6}$ & $\mathbf{0 . 0 2 0}$ & $\mathbf{0 . 0 2 6}$ & 0.982 & 0.946 & 1.000 \\
transverse AV & $\mathbf{1 . 6 9 4}$ & $\mathbf{1 . 9 7 4}$ & $\mathbf{2 . 3 1 1}$ & 0.287 & 0.333 & 0.039 \\
\hline Pelvis peak vertical & 0.144 & $\mathbf{0 . 0 0 2}$ & $\mathbf{0 . 0 0 0}$ & 0.389 & $\mathbf{0 . 0 1 3}$ & 0.662 \\
LV & 0.847 & $\mathbf{1 . 5 2 7}$ & $\mathbf{1 . 9 5}$ & 0.718 & $\mathbf{1 . 1 5}$ & 0.436 \\
\hline COM vertical LD & 0.316 & 0.197 & $\mathbf{0 . 0 4 3}$ & 0.993 & 0.122 & 0.425 \\
\hline COM peak vertical & 0.977 & 1.167 & $\mathbf{1 . 7 5 1}$ & 0.202 & 0.825 & 0.633 \\
\hline LA & 0.122 & 0.136 & $\mathbf{0 . 0 2 5}$ & 1.00 & 0.100 & $\mathbf{0 . 0 4 3}$ \\
\hline Cephalad foot peak & 1.529 & 1.487 & $\mathbf{2 . 4 6 3}$ & 0.058 & 0.926 & $\mathbf{0 . 9 8 7}$ \\
\hline GRFV & 0.291 & $\mathbf{0 . 0 5 0}$ & $\mathbf{0 . 0 0 9}$ & 0.655 & $\mathbf{0 . 0 1 3}$ & 0.400 \\
\hline Cephalad foot peak & 0.845 & $\mathbf{1 . 3 8 0}$ & $\mathbf{1 . 9 8 7}$ & 0.532 & $\mathbf{1 . 1 4 9}$ & 0.618 \\
\hline GRF AP & 0.762 & $\mathbf{0 . 0 1 1}$ & $\mathbf{0 . 0 0 9}$ & 0.128 & 0.125 & 1.000 \\
\hline Caudad foot min & 0.473 & $\mathbf{1 . 3 1 0}$ & $\mathbf{1 . 2 0 5}$ & 0.859 & 0.849 & 0.107 \\
\hline GRF & $\mathbf{0 . 0 0 0}$ & $\mathbf{0 . 0 0 0}$ & $\mathbf{0 . 0 0 0}$ & 0.998 & 0.528 & 0.298 \\
\hline Cephalad foot peak & $\mathbf{1 . 9 7 3}$ & $\mathbf{1 . 6 9 2}$ & $\mathbf{1 . 6 1 8}$ & 0.170 & 0.484 & 0.590 \\
\hline GRF unloading rate & 1.108 & 0.243 & 0.104 & 0.995 & 0.264 & 0.534 \\
\hline Caudad foot peak & $\mathbf{0 . 0 1 8}$ & $\mathbf{0 . 0 3 9}$ & $\mathbf{0 . 0 0 9}$ & 0.997 & 0.791 & 0.462 \\
GRFv unloading rate & 1.687 & 1.205 & $\mathbf{3 . 1 0 5}$ & 0.174 & 0.418 & 0.512 \\
\hline
\end{tabular}

250 Abbreviations: AV, angular velocity. LV, linear velocity. LD, linear displacement. LA,

251 linear acceleration, GRFv, vertical ground reaction force, $\mathrm{GRF}_{\mathrm{AP}}$, anterior-posterior

252 ground reaction force

253

254 TABLE 2. Post-hoc comparisons showing adjusted p-values and unbiased effect sizes

255 (italicized). Bold font indicates significant group differences.

256

257 Kinetic analysis

258 See Appendix 2 for all between group omnibus (F-test) statistics and Table 2 for post-

259 hoc group comparisons. Ground reaction force data from the caudad foot of one

260 resident were excluded due to the participant's heel landing outside the area of the

261 force plate during the thrust. 
262 Exemplar vertical (GRFv) and horizontal GRF data from one expert and one student are 263 shown in Figure 1.

264 Ground reaction forces at the beginning of the thrust

265 For GRFv of the cephalad foot at the beginning of the thrust phase there was a

266 significant difference between groups. Experts started the thrust with significantly higher

267 GRFv under the cephalad foot compared with third year and first year students, and

268 residents had higher GRFv under the cephalad foot than first year students (Table 2).

269 There was no difference between groups for GRFv of the back foot at the beginning of

270 the manipulation.

271 At the beginning of the thrust there was a group difference in the magnitude of GRFAP of

272 the cephalad foot. Experts had larger, more positive GRFAP than third year students and

273 first year students (Table 2). There was no difference between groups for $\mathrm{GRF}_{\mathrm{AP}}$ of the

274 caudad foot and GRFML forces for either foot at the beginning of the thrust.

275 During the thrust

276 During the thrust, experts demonstrated lower minimum GRFv and greater rate of

277 unloading in the caudad foot compared with the other groups (Table 2). The minimum

278 GRFv under the cephalad foot did not differ between groups and although the rate of

279 GRFv unloading under the cephalad foot was significantly different across groups there 280 were no significant pairwise comparisons (Table 2).

281 Force-force analyses 
282 For the force-force analyses, an additional participant from the first year student group was excluded due to loss of force plate data. The force-force analyses demonstrated

284 that there were three primary patterns of ground reaction force coordination between

285 feet during the thrust: inphase decreasing (GRFv decreasing under both feet at the 286 same rate), cephalad foot decreasing, and caudad foot decreasing. Across the groups,

287 thirty-four individuals utilized predominantly inphase decreasing coordination, six 288 individuals demonstrated predominantly cephalad foot decreasing coordination and one 289 individual demonstrated predominantly caudad foot decreasing coordination. There was 290 no difference between groups for the percentage of time spent in any of the 291 coordination patterns during the manipulation.

292 Within the subgroup of participants who primarily utilized the inphase decreasing 293 coordination strategy however, there was a trend toward a group difference. Experts 294 tended to spend a greater percentage of thrust time inphase than residents and third 295 year students.

297 Regression analysis

298 Peak downward linear velocity of the pelvis was selected as the manipulation 299 performance metric for the regression analysis. Variables that were significantly 300 associated with downward pelvis velocity and were included in the regression model are 301 shown in Appendix 3.: In addition to years of experience, peak downward velocity of the 302 pelvis during the thrust was predicted best by a combination of COM displacement and $303 \mathrm{GRF}_{\mathrm{v}}$ on the cephalad foot at the beginning of the thrust (full model with 3 variables $\mathrm{R}^{2}$ $\left.304=0.668, F_{3,36}=24.107, p=.000\right)$. 


\section{DISCUSSION}

For the first time, this study demonstrates the kinematic and kinetic characteristics that delineate expert performance from more novice performance of lumbar manipulations.

The kinematic results demonstrated significant differences between expert and novice performance. The experts performed the manipulation with significantly greater downward COM acceleration. This finding is consistent with results from the Delphi study examining lumbar manipulation, with clinicians agreeing that "dropping the body downward" is an important aspect of manipulation. ${ }^{18}$ Additionally, the experts displayed faster pelvic rotation in the transverse plane, and interestingly, in the opposite direction as the other groups. Though the total arc of motion of the pelvis in transverse plane rotation is not large (approximately 7 degrees), it was different in experts compared to all other groups. Cephalad rotation of the pelvis may help to improve the force application of the experts' thrust. The close pelvis-to-pelvis contact between the therapist and the patient may allow the therapist's pelvis to "push" the patient's pelvis in a superior-anterior direction (in reference to the patient's body), providing additional force to the patient into lumbar rotation. The extent of COM vertical displacement also differed between groups. Nearly all participants elevated the COM just prior to the thrust (see Figure 2, bottom). Contemporary instruction of lumbar manipulation advises against this because it is thought that novice therapists may "de-rotate" the patient as they raise up prior to thrusting, losing joint localization. More experienced therapists are likely able to move their own COM prior to the thrust without moving the patient and thus optimize their ability to generate quick downward motion without losing the segment localization and pre-positioning. 
330 The kinetic results also demonstrated significant differences between experts and other

331 groups. First, overall increased modulation of GRFv was found in experts: they began

332 the manipulation with greater weight on the front foot, demonstrated lower minimum

333 GRFv during the manipulation, and achieved the highest rate of unloading of the back

334 foot during the manipulation. These results generally show that the experts utilize

335 vertical ground reaction forces significantly more than other groups. Again, this result

336 mirrors the results of O'Donnell et al., with clinicians agreeing that the thrust force

337 "should be generated by the body and legs", not the force applicator (usually the arm or

338 hand). ${ }^{18}$ The differences in kinetics demonstrate that the experts and more experienced

339 therapists indeed do this. The force-force results between force plates did not

340 demonstrate any significant pattern differentiation between experience levels. Most

341 participants were categorized into "inphase decreasing". This is likely due to the fact

342 that the overall movement dynamics of this technique are that the therapist is dropping

343 his body weight, and GRF must decrease in both feet.

345 Many of the factors identified in the results were not only significantly greater in experts,

346 but the means of each group formed a graduated spectrum in which, with each

347 successive increase in group experience, the group mean became more "expert like".

348 Both kinematic and kinetic results demonstrate this stepwise improvement. For

349 example, peak downward linear pelvic velocity increased significantly from first years to

350 residents, third years to experts, and first years to experts. These results are similar to

351 those found by Descarreaux in which students and clinicians of increasing experience 
352 level displayed a stepwise improvement in unloading time and hand-body delay (factors

353 which identify the quickness of the manipulation). ${ }^{8}$ Similar differences in manipulation

354 speed and force production between students and more experienced manipulators are

355 demonstrated with this stepwise improvement in other chiropractic literature. . $^{6,8,9}$

356 Although the kinetic measurement methods between this study and the other

357 chiropractic studies are different, a single similarity is seen across all studies: more

358 experienced manipulators apply force over a shorter period of time compared to

359 novices.

360

361 Since there is no previous biomechanical analysis of performance of this manipulation,

362 we must assume that the expert performance is the gold-standard. This assumption is a

363 reality in many instances of sports and performance where kinetic and kinematic norms

364 have not been established. In the data analysis, peak downward linear pelvic velocity

365 was the variable that best distinguished amongst the groups, so this was chosen as the

366 metric of manipulation performance for the regression analysis. The regression analysis

367 found peak vertical velocity of the pelvis during the thrust was predicted best by three

368 factors; normalized vertical displacement of the COM, initial GRF $v$ under the cephalad

369 foot, and years of experience. This suggests that focusing on downward COM motion

370 and loading on the cephalad foot may help to improve manipulation performance in

371 novice therapists. These are simple verbal instructions that could be given in laboratory

372 practice environments in both entry-level and continuing education curricula. 
374 There were limitations to the study. The expert group had fewer participants than the 375 resident and student groups. This was due to our efforts to recruit individuals for the

376 expert group that are leaders in the field. All students enrolled in the study were from

377 the same institution. This ensured that each group had learned the manipulation in the 378 same way, however it is not known if students from a different institution would display 379 the same results. Though we could not measure reaction forces through the table, we 380 must assume that the downward force produced by dropping the COM is being 381 transmitted through the therapists' arms, and perhaps pelvis as well. Different patient 382 models were used during the study, whose anthropometrics were not all matched, and 383 the argument may be made that two patients of different size may require different 384 magnitude of force from the practitioner. This was controlled as much as possible by 385 having each participant manipulate two different patient models and averaging the data 386 for each participant across the four trials. Finally, we did not attempt to characterize if 387 the expert-performed manipulation is a more effective/therapeutic manipulation than 388 one performed by a student. There are a multitude of factors that affect the patient389 therapist therapeutic relationship and will alter the likelihood of an intervention providing 390 the intended result.

392 CONCLUSION

393 The kinetics and kinematics of side-lying lumbar manipulation change significantly with 394 increasing practitioner experience. This study demonstrates important biomechanical 395 factors for performance of lumbar manipulation and provides information for educators 396 teaching this complex manual skill. 
398 
399 HIGHLIGHTS

400 - Operator mechanics of lumbar manipulation (SLM) are not well understood

401 - Experts rotate their pelvis in the opposite direction during SLM

402 - Experts perform SLM with greater downward pelvic velocity

403 - Pelvic velocity can be predicted though years of experience, among other factors

404

405 


\section{REFERENCES}

1. Cagnie B, Vinck E, Beernaert A, Cambier D. How common are side effects of spinal manipulation and can these side effects be predicted? Man Ther. 2004;9(3):151-156. doi:10.1016/j.math.2004.03.001

2. Cambridge EDJ, Triano JJ, Ross JK, Abbott MS. Comparison of force development strategies of spinal manipulation used for thoracic pain. Man Ther. 2012;17(3):241-245. doi:10.1016/j.math.2012.02.003

3. Chandran Suja V, Barakat Al. A Mathematical Model for the Sounds Produced by Knuckle Cracking. Sci Rep. 2018;8(1):1-9. doi:10.1038/s41598018-22664-4

4. Chang J-Y, Chang G-L, Chang Chien C-J, Chung K-C, Hsu A-T. Effectiveness of two forms of feedback on training of a joint mobilization skill by using a joint translation simulator. Phys Ther. 2007;87(4):418-430. doi:10.2522/ptj.20060154

5. Childs MJD, Fritz JM, Flynn TW, et al. A clinical prediction rule to identify patients with low back pain most likely to benefit from spinal manipulation: A validation study. Ann Intern Med. 2004;141(12):920-928. doi:141/12/920 [pii]

6. Cohen E, Triano JJ, McGregor M, Papakyriakou M. Biomechanical Performance of Spinal Manipulation Therapy by Newly Trained vs. Practicing Providers: Does Experience Transfer to Unfamiliar Procedures? Vol 18.; 1995.

7. Descarreaux M, Dugas C, Raymond J, Normand MC. Kinetic analysis of expertise in spinal manipulative therapy using an instrumented manikin. $J$ Chiropr Med. 2005;4(2):53-60. doi:10.1016/S0899-3467(07)60114-1

8. Descarreaux M, Dugas C. Learning Spinal Manipulation Skills: Assessment of Biomechanical Parameters in a 5-Year Longitudinal Study. J Manipulative Physiol Ther. 2010;33(3):226-230. doi:10.1016/j.jmpt.2010.01.011

9. DeVocht JW, Owens EF, Gudavalli MR, Strazewski J, Bhogal R, Xia T. Force-time profile differences in the delivery of simulated toggle-recoil spinal manipulation by students, instructors, and field doctors of chiropractic. $J$ Manipulative Physiol Ther. 2013;36(6):342-348. doi:10.1016/j.jmpt.2013.05.027

10. Dunnett CW, Dunnett CW. in the Unequal Pairwise Multiple Comparisons Variance Case. J Am Stat Assoc. 2012;75(372):796-800.

11. Flynn T, Fritz J, Whitman J, et al. A Clinical Prediction Rule for classifying patients with low back pain who demonstrate short-term improvement with spinal manipulation. Spine (Phila Pa 1976). 2002;27(24):2835-2843.

doi:10.1097/00007632-200212150-00021

12. Fritz CO, Morris PE, Richler JJ. Effect size estimates: Current use, calculations, and interpretation. J Exp Psychol Gen. 2012;141(1):2-18. doi:10.1037/a0024338 
13. Gard SA, Miff SC, Kuo AD. Comparison of kinematic and kinetic methods for computing the vertical motion of the body center of mass during walking. Hum Mov Sci. 2004;22(6):597-610. doi:10.1016/j.humov.2003.11.002

14. Jull G, Moor A, Falla D, Lewis J, McCarthy CJ, Sterling M. Grieve's Modern Musculoskeletal Physiotherapy. 4th ed. Elsevier; 2015.

15. Koes BW, van Tulder M, Lin C-WC, Macedo LG, McAuley J, Maher C. An updated overview of clinical guidelines for the management of non-specific low back pain in primary care. Eur Spine J. 2010;19(12):2075-2094. doi:10.1007/s00586-010-1502-y

16. Koppenhaver SL, Hebert JJ, Kawchuk GN, et al. Criterion validity of manual assessment of spinal stiffness. Man Ther. 2014;19(6):589-594. doi:10.1016/j.math.2014.06.001

17. Needham R, Naemi R, Chockalingam N. Quantifying lumbar-pelvis coordination during gait using a modified vector coding technique. J Biomech. 2014;47(5):1020-1026. doi:10.1016/j.jbiomech.2013.12.032

18. O'Donnell M, Armour Smith J, Abzug A, et al. How Should We Teach Lumbar Manipulation? A Consensus Study. Man Ther. 2016;25:1-10. doi:10.1017/CBO9781107415324.004

19. Pillastrini $P$, Gardenghi I, Bonetti $F$, et al. An updated overview of clinical guidelines for chronic low back pain management in primary care. Jt Bone Spine. 2012;79(2):176-185. doi:10.1016/j.jbspin.2011.03.019

20. Rubinstein SM, Middelkoop M Van, Boer MR De, et al. Spinal Manipulative Therapy for Chronic Low-Back Pain. Spine (Phila Pa 1976). 2011;36(2):2-5. doi:10.1097/BRS.0b013e3182197fe1

21. Triano JJ, Gissler T, Forgie M, Milwid D. Maturation in rate of high-velocity, low-amplitude force development. J Manipulative Physiol Ther. 2011;34(3):173-180. doi:10.1016/j.jmpt.2011.02.007

22. Van Geyt B, Dugailly PMA, De Page L, Feipel V. Relationship Between Subjective Experience of Individuals, Practitioner Seniority, Cavitation Occurrence, and 3-Dimensional Kinematics During Cervical Spine Manipulation. J Manipulative Physiol Ther. 2017;40(9):643-648. doi:10.1016/j.jmpt.2017.09.002

23. Vos T, Allen C, Arora M, et al. Global, regional, and national incidence, prevalence, and years lived with disability for 310 diseases and injuries, 1990-2015: a systematic analysis for the Global Burden of Disease Study 2015. Lancet. 2016;388(10053):1545-1602. doi:10.1016/S01406736(16)31678-6 